\title{
Intoxicação espontânea por Amaranthus hybridus (Amaranthaceae) em bovinos no sul do Rio Grande do Sul ${ }^{1}$
}

\author{
Adriana Lücke Stigger ${ }^{2}$, Clairton Marcolongo-Pereira², Maria de Lourdes Adrien², \\ Bianca L. Santos ${ }^{3}$, Leticia Fiss ${ }^{2}$, Sergio F. Vargas Jr. ${ }^{2}$, Fabiane Borelli Grecco ${ }^{4}$ \\ e Ana Lucia Schild ${ }^{5 *}$
}

\begin{abstract}
Stigger A.L., Marcolongo-Pereira C., Adrien M.L., Santos B.L., Fiss L., Vargas Jr S.F., Grecco F.B. \& Schild A.L. 2013. [Spontaneous poisoning by Amaranthus hybridus in cattle in southern Rio Grande do Sul.] Intoxicação espontânea por Amaranthus hybridus (Amaranthaceae) em bovinos no sul do Rio Grande do Sul. Pesquisa Veterinária Brasileira 33(8):1004-1008. Laboratório Regional de Diagnóstico, Faculdade de Veterinária, Universidade Federal de Pelotas, Campus Universitário s/n, Centro, Pelotas, RS 96010-900, Brazil. E-mail: alschild@terra.com.br

An outbreak of Amaranthus hybridus poisoning in cattle was observed in February/March 2013 in southern Rio Grande do Sul, Brazil. The morbidity rate observed was 48.33\%, the mortality rate was $41.66 \%$ and lethality rate was $86.20 \%$. The diagnosis of $A$. hybridus poisoning was made based on the epidemiological data, clinical signs, macroscopic and histological lesions characteristic of intoxication by nephrotoxic plants and by the presence of large amounts of the plant in the area where cattle were placed. Invasion of Brachiaria sp. pasture by A. hybridus alerts for the possibility of the occurrence of poisoning not only in stubble areas with lack of forage. In the case reported here there was abundant forage availability in the area where the outbreak occurred. Favorable climatic conditions and fertile soil for planting pasture, probably favored the growth of this invasive species.
\end{abstract}

INDEX TERMS: Poisonous plants, Amaranthus hybridus, Amaranthaceae, nephrotoxic plants, plant poisoning, cattle.

RESUMO.- Descreve-se um surto de intoxicação por Amaranthus hybridus ocorrido em bovinos em fevereiro/março de 2013 na região sul do Rio Grande do Sul. A morbidade foi de $48,33 \%$, a mortalidade de $41,66 \%$ e a letalidade 86,20\%. 0 diagnóstico da intoxicação por $A$. hybridus foi baseado nos dados epidemiológicos, sinais clínicos, achados de necropsia e lesões histológicas características da intoxicação por plantas nefrotóxicas e pela presença da planta em grande quantidade na área onde estavam os bovinos.

\footnotetext{
${ }^{1}$ Recebido em 17 de julho de 2013.

Aceito para publicação em 9 de agosto de 2013.

${ }^{2}$ Pós-Graduanda em Veterinária, Faculdade de Veterinária (FV), Universidade Federal de Pelotas (UFPel), Campus Universitário s/n, Pelotas, RS 96015-560, Brasil.

${ }^{2}$ Médica Veterinária, Bolsista de Apoio Técnico - CNPq/INCT, Proc. 573534/2008-0, Laboratório Regional de Diagnóstico (LRD), FV-UFPel, Campus Universitário s/n, Pelotas, RS.

${ }^{4}$ Docente do Departamento de Patologia Animal, FV-UFPel, Campus Universitário s/n, Pelotas, RS.

${ }^{5}$ Laboratório Regional de Diagnóstico, FV-UFPel. *Autor para Correspondência: alschild@terra.com.br
}

A invasão da pastagem de Brachiaria sp. pela planta alerta para a possibilidade da ocorrência da intoxicação não somente em áreas de resteva com carência de forragem, uma vez que no surto relatado neste trabalho havia abundante disponibilidade de forragem nas áreas onde os bovinos se intoxicaram. No presente caso provavelmente as boas condições climáticas e o solo fertilizado para a plantação da pastagem favoreceram o crescimento da invasora.

TERMOS DE INDEXAÇÃO: Plantas tóxicas, caruru, Amaranthus hybridus, Amaranthaceae, plantas nefrotóxicas, intoxicação por plantas, bovinos.

\section{INTRODUÇÃo}

Diversas espécies de Amaranthus (caruru) têm sido relatadas como tóxicas para bovinos, causando nefrose tubular, como Amaranthus hybridus, A. thus blitum, A. spinosus, $A$. quitensis e A. retroflexus (Cursack \& Romano 1967, Stuart et al. 1975, Ferreira et al. 1991, Lemos et al. 1993). A intoxicação ocorre, também, em outras espécies animais como suínos pela ingestão de Amaranthus caudatus, A. viridis, $A$. 
quitensis e A. retroflexus, (Buck et al. 1966, Kommers et al. 1996 ) e em ovinos causada por $A$. spinosus (Peixoto et al. 2003).

A intoxicação por Amaranthus spp. foi descrita em bovinos no Rio Grande do Sul associada a ingestão de grandes quantidades da planta que invadiam lavouras não colhidas ou colhidas parcialmente com severa carência de forragem devido a estiagem no verão ou no outono (Ferreira et al. 1991, Lemos et al. 1993, Torres et al. 1997). Amaranthus spp. são descritas como plantas palatáveis e consumida normalmente pelos animais (Riet-Correa et al. 1983, Ferreira et al. 1991). É mencionado, também, que a intoxicação é rara ocorrendo em áreas invadidas pela planta, quando os bovinos estão com fome e são colocados em altas lotações nas áreas invadidas (Torres et al. 1997). A morbidade nos surtos pode variar de 5\%-40\% e a letalidade é alta, podendo chegar a 100\% (Ferreira et al. 1991, Lemos et al. 1993). O princípio ativo responsável pela ação nefrotóxica de Amaranthus spp. não é conhecido (Riet-Correa \& Méndez 2007).

A intoxicação provoca nefrose tubular tóxica que, em geral, cursa com edemas, sobretudo perirrenal, por vezes associado à presença de cristais de oxalatos nos túbulos uriníferos. A evolução desse quadro é de 7-14 dias. Experimentalmente, a administração para bovinos de $A$. blitum na dose de 492,25g/kg de peso corporal durante 10 dias causou lesões histológicas discretas de nefrose (Ferreira et al. 1991). A intoxicação por nitratos/nitritos decorrente da ingestão de Amaranthus spp. foi diagnosticada presuntivamente em uma resteva de milho (Schild et al. 1996).

O objetivo deste trabalho foi descrever os aspectos epidemiológicos e clínico-patológicos de um surto de intoxicação espontânea por $A$. hybridus que ocorreu em uma pastagem de Brachiaria spp. com boa disponibilidade de forragem na região sul do Rio Grande do Sul.

\section{MATERIAL E MÉTODOS}

Os encéfalos de dois bovinos que apresentaram sinais neurológicos e morreram em uma propriedade localizada na região sul do Rio Grande do Sul foram remetidos ao Laboratório Regional de Diagnóstico (LRD) da Universidade Federal de Pelotas (UFPel) com suspeita de raiva, já que, em uma propriedade próxima havia sido confirmada a ocorrência desta enfermidade em bovinos no mesmo período. Como o resultado do exame para esta enfermidade foi negativo a propriedade foi visitada pelos técnicos do LRD/ UFPel sendo investigados os dados epidemiológicos e sinais clínicos e necropsiados cinco bovinos (Bovinos 1, 2 3, 4 e 5) recém mortos ou eutanasiados in extremis, os quais apresentavam os mesmos sinais clínicos dos dois primeiros bovinos mortos. Fragmentos de todos os órgãos, incluindo o sistema nervoso central foram fixados em formalina $10 \%$, processados rotineiramente e corados com hematoxilina e eosina (HE). Foi realizada coleta de sangue de 14 bovinos do lote, dos quais sete, apresentavam sinais clínicos da doença, para realização da bioquímica sérica. As áreas onde os animais estavam foram percorridas para observação da presença de plantas tóxicas que pudessem causar o quadro clínico apresentado pelos animais.

\section{RESULTADOS}

O surto foi observado entre fevereiro e março de 2013 em uma propriedade do município do Capão do Leão, RS (La- titude $31^{\circ} 48^{\prime} 21.7^{\prime \prime}$ S e Longitude $52^{\circ} 31^{\prime} 58.3^{\prime \prime}$ W), em duas áreas de pastagem de Brachiaria sp., plantadas em julho de 2012 e severamente invadidas por Amaranthus hybridus (Pel 26204) que no momento do surto estava em fase de floração e da mesma altura da braquiária (Fig.1). De um total de 60 bovinos com idades entre 1-6 anos, que tinham sido adquiridos em janeiro de 2013, 29 adoeceram e 25 morreram 20-30 dias após o ingresso na pastagem. As áreas da pastagem tinham entre 4 e 7 hectares e estavam com excelente disponibilidade de forragem (Fig.1) e os bovinos permaneceram aproximadamente 15 dias em cada área. A morbidade foi de $48,3 \%$ e a letalidade foi $86,2 \%$. Quatro animais se recuperaram em aproximadamente 20 dias.

Além dos sinais neurológicos de incoordenação motora e, em alguns casos agressividade, observados pelo veterinário da propriedade, os bovinos apresentavam tremores musculares, perda de equilíbrio, dificuldade de locomoção, emagrecimento progressivo (Fig.2), desidratação e diarreia escura ou com sangue. A evolução do quadro clínico foi em média 3-4 dias. As concentrações de ureia e creatinina sérica avaliadas em 14 bovinos do lote são apresentadas no Quadro 1.

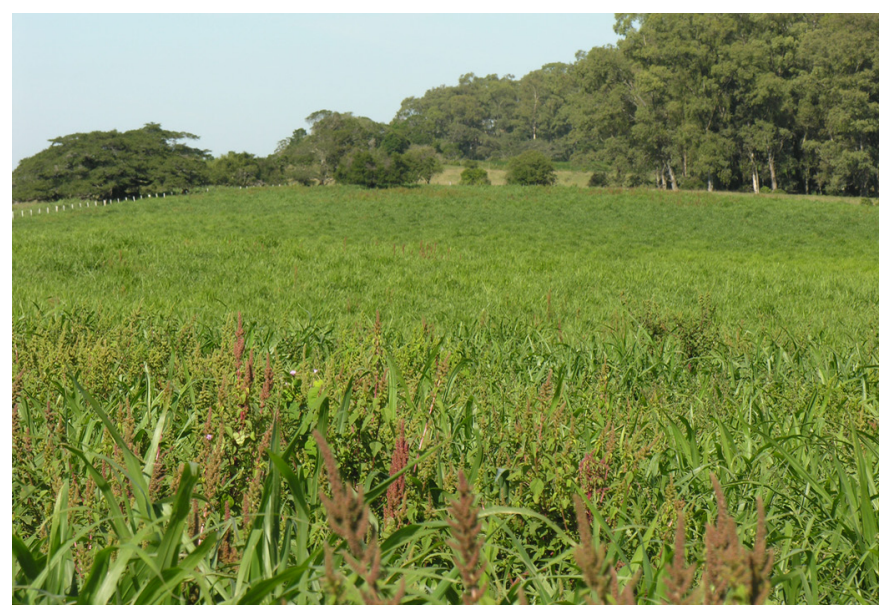

Fig.1. Pastagem de Brachiaria sp. severamente invadida por Amaranthus hybridus que está em frutificação e na mesma altura da pastagem.

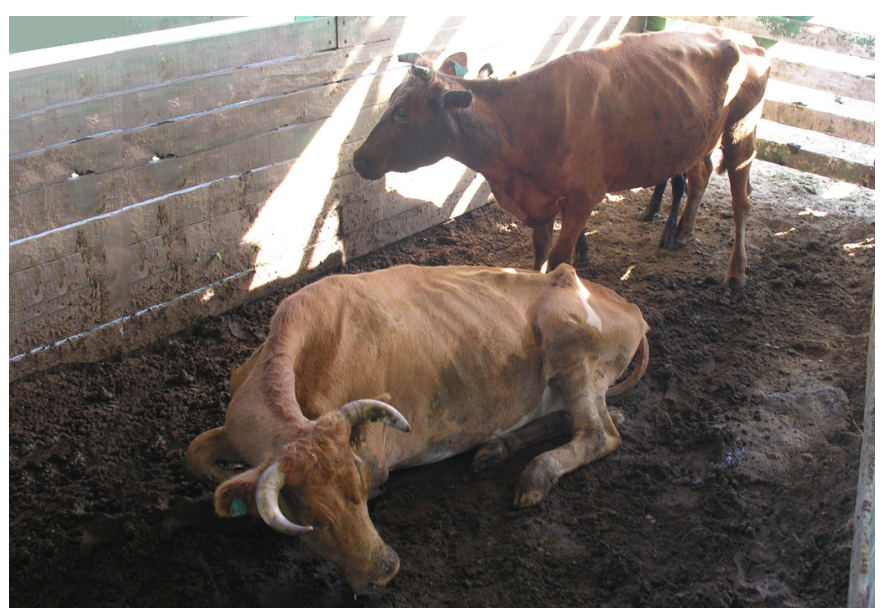

Fig.2. Bovinos intoxicados por Amaranthus hybridus apresentando emagrecimento e apatia. 
Quadro 1. Concentração de ureia e creatinina no soro de 14 bovinos sob risco de intoxicação por Amaranthus hybridus

\begin{tabular}{cccccc}
\hline Bovino & $\begin{array}{c}\text { Ureia }^{\mathrm{a}} \\
\mathrm{mg} / \mathrm{dL}\end{array}$ & $\begin{array}{c}\text { Creatinina }^{\mathrm{b}} \\
\mathrm{mg} / \mathrm{dL}\end{array}$ & Bovino & $\begin{array}{c}\text { Ureia } \\
\mathrm{mg} / \mathrm{dL}\end{array}$ & $\begin{array}{c}\text { Creatinina } \\
\mathrm{mg} / \mathrm{dL}\end{array}$ \\
\hline 1 & 33 & 1.47 & 8 & 11 & 1,38 \\
2 & 18 & 0,89 & 9 & 29 & 1.5 \\
3 & 11 & 0,99 & 10 & 16 & 1.05 \\
4 & 32 & 1.25 & 11 & 19 & 1.07 \\
5 & 18 & 1.0 & 12 & 17 & 1.01 \\
6 & 23 & 1.37 & 13 & 207 & 11.34 \\
7 & 17 & 1,13 & 14 & 601 & 14.75 \\
\hline
\end{tabular}

$\overline{{ }^{a}}$ Valor de referência $=6-22 \mathrm{mg} / \mathrm{dL} ;{ }^{b}$ valor de referência $=0,5-1,10 \mathrm{mg} / \mathrm{dL}$.

Macroscopicamente havia ascite, edema do mesentério e edema perirrenal hemorrágico (Fig.3A), que eram mais acentuados nos Bovinos 2, e 3 e 4 . Havia erosões e úlceras no esôfago e abomaso (Fig.3B), petéquias nas serosas e congestão da mucosa do reto que continha fezes secas e escuras recobertas por muco e estrias de sangue no Bovino 4. Os rins eram acentuadamente pálidos nos cinco bovinos, tanto na superfície capsular (Fig.4A) como na de corte (Fig. 4B) e havia edema da região do hilo.

As lesões histológicas, embora semelhantes em todos os bovinos afetados, variavam quanto a intensidade e eram caracterizadas por degeneração e necrose das células epiteliais dos túbulos renais, associadas à presen-
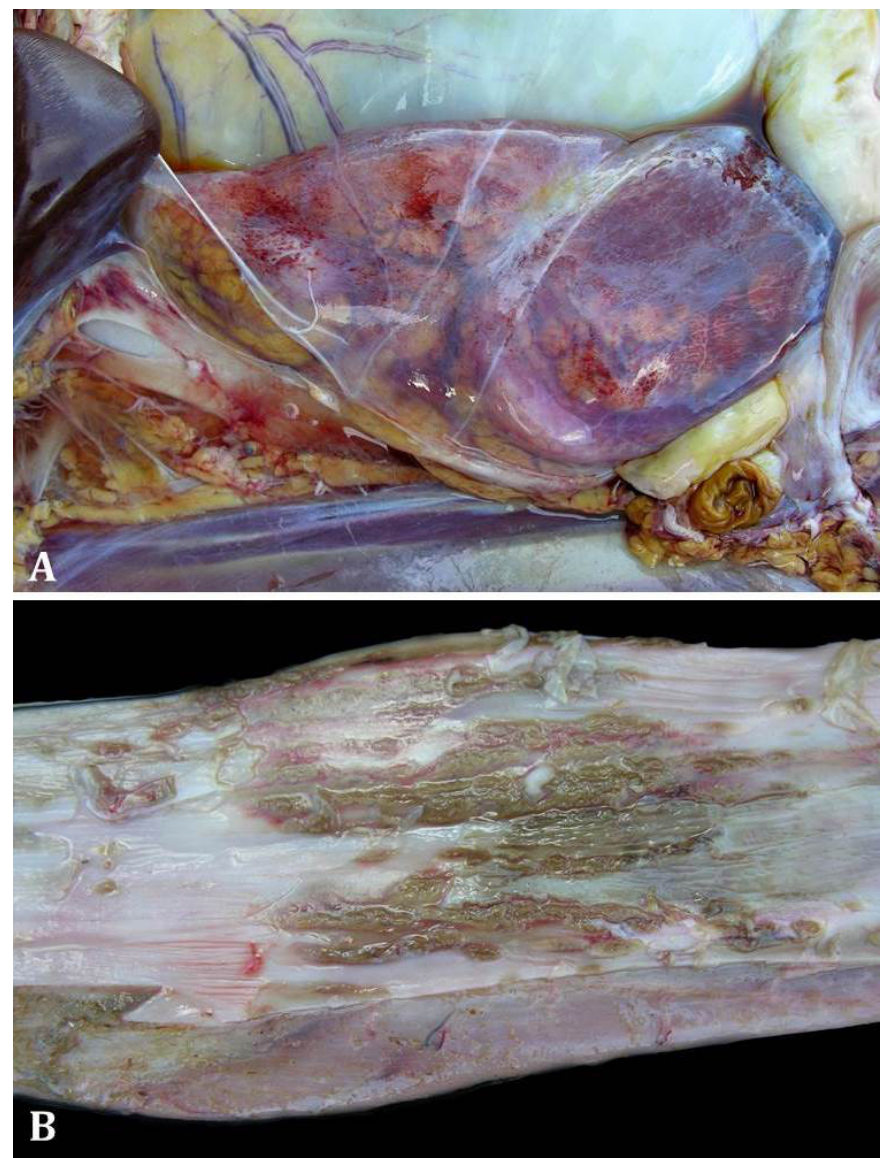

Fig.3. (A) Rim de bovino intoxicado por Amaranthus hybridus com edema perirrenal hemorrágico acentuado. (B) Esôfago de bovino intoxicado por Amaranthus hybridus apresentando erosões e úlceras na mucosa.

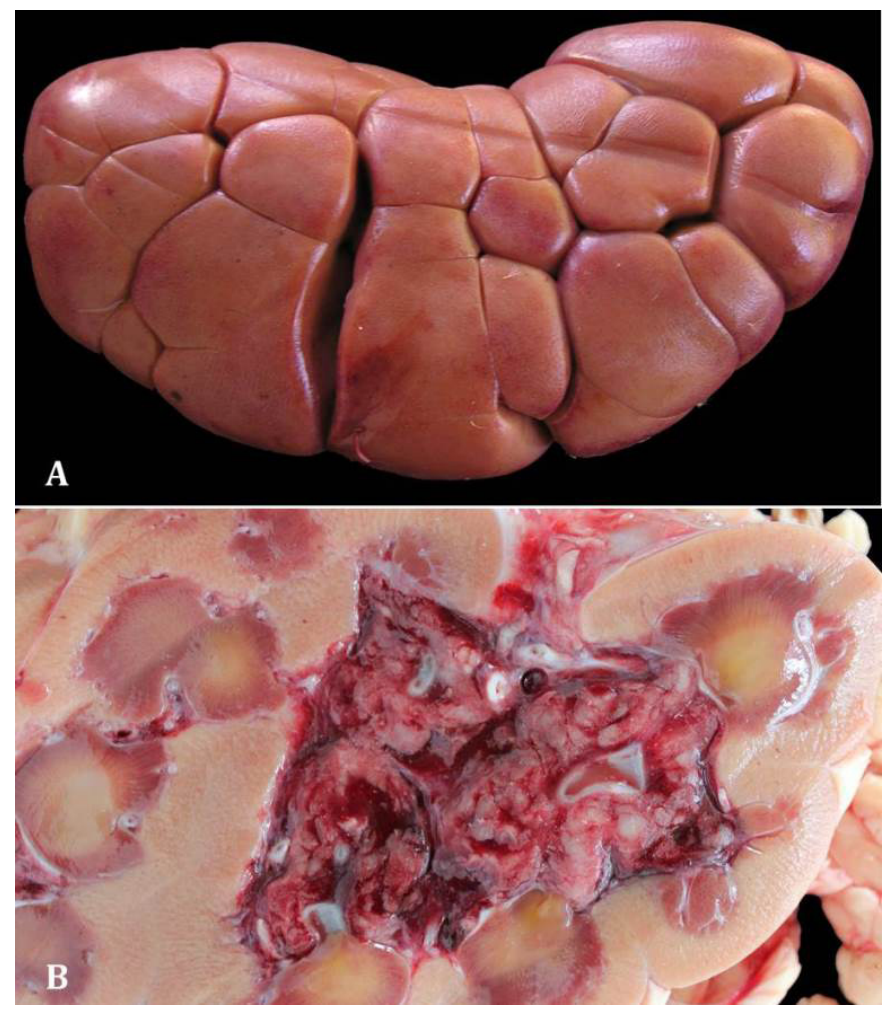

Fig.4. (A) Superfície capsular do rim de bovino intoxicado por Amaranthus hybridus apresentando palidez acentuada. (B) Superfície de corte do rim de bovino intoxicado por Amaranthus hybridus pálido e com edema hemorrágico na região da pélvis.

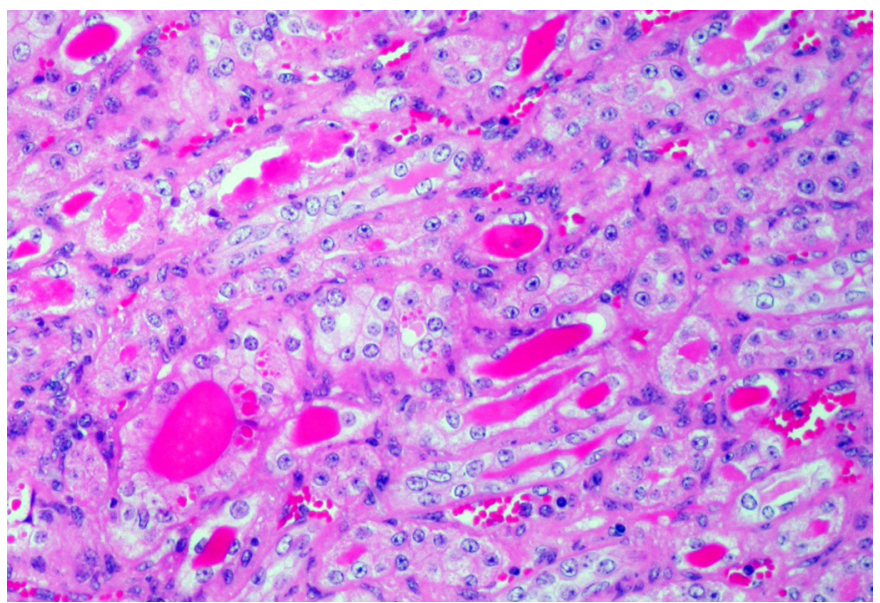

Fig.5. Corte histológico de rim de bovino intoxicado por Amaranthus hybridus apresentando degeneração do epitélio tubular e presença de cilindros hialinos no interior dos túbulos.

ça de cilindros hialinos na luz tubular (Fig.5), acúmulo de material eosinofílico nos glomérulos e espessamento da cápsula de Bowman. No esôfago havia necrose de coagulação na superfície epitelial, com perda celular associada a intenso infiltrado de neutrófilos que invadia a submucosa. Nos demais órgãos, incluindo o sistema nervoso central, não havia lesões de significado patológico; tampouco foram encontrados cristais de oxalato nos rins e no sistema nervoso central de todos os cinco bovinos necropsiados. 


\section{DISCUSSÃO}

No presente estudo o diagnóstico foi realizado com base nos dados epidemiológicos, sinais clínicos, achados de necropsia, lesões histológicas e pelos altos níveis de ureia e creatinina encontrados no soro sanguíneo de bovinos afetados e, ainda, pela presença de Amaranthus hybridus em grande quantidade na área onde os animais adoeceram.

Tem sido descrito que a intoxicação por Amaranthus spp. não é frequente e os surtos ocorrem somente quando há escassez de forragem em presença de grande quantidade da planta (Cursack \& Romano 1967, Stuart et al. 1975; Ferreira et al. 1991, Lemos et al. 1993, Torres et al. 1997, Tokarnia et al. 2012).

Diversos autores mencionam que Amaranthus spp. é uma planta palatável (Riet-Correa et al. 1983, Ferreira et al. 1991, Pessoa et al. 2013), o que provavelmente influenciou na ocorrência do surto aqui relatado. Chama a atenção que no presente surto não havia escassez de forragem e, aparentemente os bovinos ingeriram a planta misturada à braquiária em quantidade suficiente para se intoxicar.

É mencionado que Amaranthus spp. é uma planta invasora que cresce bem em solos férteis e áreas adubadas e por isso frequentemente invade lavouras, que após a colheita são utilizadas para a alimentação do gado (Tokarnia et al. 2012).

No Rio Grande do Sul os surtos descritos ocorreram tanto no outono (Ferreira et al. 1991) como no verão (Ferreira et al. 1991, Lemos et al. 1993, Schild et al. 1996, Torres et al. 1997). Em todos estes surtos Amaranthus spp. estava em estágio de frutificação e encontrava-se invadindo restevas de culturas como sorgo, soja, milho ou melancia, não colhidas ou parcialmente colhidas. 0 surto relatado neste trabalho ocorreu no verão, com a planta, também, em estágio de frutificação, sugerindo que conforme tem sido mencionado por diversos autores (Cursack \& Romano 1967, Duffy et al. 1985, Armesto et al. 1989, Ferreira et al. 1991) este é o estágio em que as diferentes espécies de Amaranthus são tóxicas.

A invasão da pastagem de Brachiaria sp. por A. hybridus demonstra a ocorrência da intoxicação não somente em áreas de resteva com escassez de forragem. No presente caso, provavelmente, as boas condições climáticas e o solo fertilizado para a plantação da pastagem favoreceram o crescimento da invasora. Outro fato que deve ser considerado é a qualidade da semente utilizada. De acordo com o proprietário a semente de braquiária utilizada nas áreas onde o surto ocorreu era certificada o que indica que Amaranthus estava presente no banco de sementes do solo e que as condições adequadas de adubação permitiram sua germinação e emergência junto com a braquiária.

A morbidade de 48,3\% observada neste surto foi similar ao encontrado por outros autores (Ferreira et al. 1991, Schild et al. 1996, Torres et al. 1997) e a letalidade foi, também, elevada $(86,2 \%)$ o que demonstra que apesar de rara, a intoxicação quando ocorre causa perdas consideráveis. Contribuiu, também, para a alta morbidade neste caso, a suspeita inicial de raiva e o envio ao laboratório somente da cabeça dos primeiros bovinos mortos o que retardou o diagnóstico correto da enfermidade. Os sinais clínicos neu- rológicos observados ocorreram em consequência da uremia (Carlson 1990).

É importante realizar o diagnóstico diferencial da intoxicação por outras plantas nefrotóxicas, como Thiloa glaucocarpa, Dimorphandra mollis e Dimorphandra gardneriana, as quais podem causar quadro clínico-patológico semelhante ao provocado por Amaranthus spp., entretanto, estas plantas não ocorrem no Rio Grande do Sul (Tokarnia et al., 2012). Setaria spp. pode causar, também, nefrose tóxica (Tokarnia et al. 2012) e ocorre na região sul do Estado, no entanto essa gramínea não estava presente nas áreas onde ocorreu o surto. Anagallis arvensis causa nefrose em bovinos no Uruguai (Rivero 1998), e ocorre em regiões alagadas no sul do Rio Grande do Sul, porém esta planta também não foi encontrada nas áreas onde os bovinos adoeceram.

As variações das lesões macroscópicas e histológicas entre os animais afetados provavelmente estão relacionadas a quantidade da planta ingerida pelos bovinos. Deve ser mencionado que edema perirrenal hemorrágico e ascite foram observados somente nos animais eutanasiados in extremis. Entretanto, a palidez renal estava presente em todos os bovinos necropsiados sugerindo que apesar de muitas vezes não haver lesões muito espetaculares durante a necropsia a lesão de insuficiência renal aguda representada pela palidez do órgão é o achado característico da intoxicação.

Não há tratamento para a forma nefrotóxica da intoxicação por Amaranthus spp. em bovinos. A única forma de evitar a intoxicação é retirar os bovinos das áreas invadidas, principalmente quando a planta está em frutificação. As lesões renais levam a uremia evidenciada pela alteração nas concentrações de ureia e creatinina no soro sanguíneo resultando nas severas erosões e ulcerações nas mucosas especialmente do esôfago e abomaso. De fato, essas alterações são semelhantes às descritas por outros autores, que encontraram níveis elevados de uréia e creatinina em bovinos intoxicados espontaneamente por Amaranthus spp. (Stuart et al. 1975, Armesto et al. 1989, Ferreira et al. 1991).

A ocorrência de intoxicações por plantas no Brasil tem sido largamente estudada sendo bem conhecidas as espécies ou gêneros que causam grandes prejuízos econômicos anuais para a pecuária (Riet-Correa \& Medeiros 2001, Pessoa et al. 2013). No entanto, algumas intoxicações apesar de não ocorrerem todos os anos podem causar prejuízos consideráveis por não serem bem conhecidas por produtores e veterinários como o caso do presente surto de intoxicação por A. hybridus.

Agradecimentos.- Trabalho financiado pelo INCT/CNPq (Proc.573534/ 2008-0).

\section{REFERÊNCIAS}

Armesto R.R., Grande H.A., Baroni A.C., Baroni E.E. \& Blogo J. 1989. Intoxicación por Amaranthus quitensis en vaquillonas Holando Argentino. Vet. Arg. 6:692-700

Buck W.V., Preston K.S., Abel M. \& Marshall V.L. 1966. Perirenal edema in swine: a disease caused by common weeds. J. Am. Med. Vet. Assoc. 148(12):1525-1531.

Carlson G.P. 1990. Clinical chemical test, p.386-414. In: Smith B.D. (Ed.), Large Animal Internal Medicine. C.V. Mosby, Baltimore. 
Cursack H.A. \& Romano L.A. 1967. Possible intoxicación sub-aguda con nitritos y nitratos. El "yuyo colorado" (Amaranthus hybridus var. quitensis) como espécie suspechosa de ser toxica para el ganado vacuno. Gaceta Vet. 29:69-74.

Duffy S.J., León E.A., Gavier M.D., Vieira F.J.B., Corbelini C.N. \& Nogueira M. 1985. Intoxicación por Amaranthus quitensis (Yuyo Colorado) en bovinos. Vet. Arg. 2:942-949.

Ferreira J.L.M., Riet-Correa F., Schild A.L. \& Méndez M.C. 1991. Intoxicação por Amaranthus spp. (Amaranthaceae) em bovinos no Rio Grande do Sul. Pesq. Vet. Bras. 11(3/4):49-54.

Kommers G.D., Torres M.B., Dantas A.F. \& Barros C.S.L. 1996. Intoxicação por Amaranthus spp (Amaranthaceae) em suínos no Rio Grande do Sul. Pesq. Vet. Bras. 16(4):121-125.

Lemos R.A., Barros C.S.L., Salles M.S., Barros S.S. \& Peixoto P.V. 1993. Intoxicação espontânea por Amaranthus spinosus (Amaranthaceae) em bovinos. Pesq. Vet. Bras. 13(1/2):25-34.

Peixoto P.V., Brust L.A.C., Brito M.F., França T.N., Cunha B.R.M. \& Andrade G.B. 2003. Intoxicação natural por Amaranthus spinosus (Amaranthaceae) em ovinos no Sudeste do Brasil. Pesq. Vet. Bras. 23(4):179-184.

Pessoa C.R.M., Medeiros R.M.T. \& Riet-Correa F. 2013. Importância econômica, epidemiologia e controle das intoxicações por plantas no Brasil. Pesq. Vet. Bras. 33(6):752-758.

Riet Correa F., Méndez M.C. \& Schild A.L. 1983. Intoxicações por Plantas e
Micotoxicoses em Animais Domésticos. Editorial Hemisfério Sul do Brasil, Pelotas, RS, Brasil. 340p.

Riet-Correa F. \& Medeiros R.M.T. 2001. Intoxicações por plantas em ruminantes no Brasil e no Uruguai: importância econômica, controle e riscos para a saúde pública. Pesq. Vet. Bras. 21(1):38-42.

Riet-Correa F. \& Méndez M.C. 2007. Intoxicações por plantas e micotoxinas, p.99-222. In: Riet-Correa F., Schild A.L., Lemos R.A.A. \& Borges J.R. (Eds), Doenças de Ruminantes e Eqǘ́deos. Vol. 2. $3^{\text {a }}$ ed. Pallotti, Santa Maria, RS.

Rivero R. 1998. Intoxicación por Anagallis arvensis en bovinos y ovinos em Uruguay. XXVI Jornadas Uruguayas de Buiatria. Paysandú, Uruguai, p.26-30.

Schild A.L., Riet-Correa F., Ruas J.L., Riet-Correa G., Fernandes C.G., Motta A., Méndez M.C. \& Soares M. 1996. Doenças diagnosticadas pelo Laboratório Regional de Diagnóstico no ano de 1995. Boletim do Laboratório Regional de Diagnóstico no 16, Pelotas. 108p.

Stuart B.P., Nicholson S.S. \& Smith J.B. 1975. Perirenal edema and toxic nephrosis in cattle, associated with ingestion of pigweed. J. Am. Vet. Assoc. 167(10):949-950.

Tokarnia C.H., Brito M.F., Barbosa J.D., Peixoto P.V. \& Döbereiner J. 2012. Plantas Tóxicas do Brasil para Animais de Produção. Editora Helianthus, Rio de Janeiro, p.211-217.

Torres M.B., Kommers G.D., Dantas A.F.M. \& Barros C.S.L. 1997. Redroot pigweed (Amaranthus retroflexus) poisoning of cattle in Southern Brazil. Vet. Hum. Toxicol. 39(2):94-96. 